\title{
Evaluation of NMU-Induced Breast Cancer Treated with Sirolimus and Sunitinib on Breast Cancer Growth
}

\author{
Nurul Fathiyatul Nabila Jaffar ${ }^{1}$, Muhammad Shahidan Muhammad Sakri', \\ Hasnan Jaafar', Wan Faiziah Wan Abdul Rahman', Tengku Ahmad Damitri \\ Al-Astani Tengku Din ${ }^{2 *}$
}

\begin{abstract}
Objective: To analyze the effect of sirolimus and sunitinib in blocking the tumor growth and to evaluate the expressions of estrogen receptor $(E R)$, progesterone receptor $(P g R)$, and human epidermal growth factor receptor-2 (HER2/neu) after treated with sirolimus and sunitinib. Methods: Thirty-two female Sprague Dawley rats at age 21-days old were administered intraperitoneally with N-Methyl-N-Nitroso Urea (NMU), dosed at 70mg/kg body weight. The rats were divided into 4 groups; Group 1 (Control, n=8), Group 2 (Sirolimus, n=8), Group 3 (Sunitinib, n=8) and Group 4 (Sirolimus+Sunitinib, $\mathrm{n}=8$ ), being treated twice when the tumor reached the size of $14.5 \pm 0.5 \mathrm{~mm}$ and subsequently sacrificed after 5 days. The protein expressions of $E R, P g R$ and HER2/neu of the tumor tissues were evaluated by using immunohistochemistry analysis. Results: Treatment with sirolimus alone lowered expressions of $E R$ and $P g R$ of breast cancer and reduced tumor size. There was no significant difference of $E R$ and $P g R$ expressions between control and sunitinib treated tumor. Sunitinib treated tumors reduce in diameter after the first treatment, however the diameter increases after the second treatment. Histologically, sunitinib treated tumor did not show any aggressive invasive carcinoma of no special type (NST) histological subtypes. In addition, all NMU-induced tumors are HER2/neu-negative scoring. Conclusion: Sirolimus is neither synergistic nor additive with sunitinib for breast cancer treatment.
\end{abstract}

Keywords: Sirolimus- sunitinib- estrogen receptor- progesterone receptor- human epidermal growth factor receptor-2

Asian Pac J Cancer Prev, 21 (10), 2919-2925

\section{Introduction}

Breast cancer is a heterogeneous disease with a wide variety of clinical, pathological, and molecular characteristics, the most commonly diagnosed cancer among females and the leading cause of women cancer death (Bray et al., 2018). According to the Malaysia National Cancer Registry Report (2019), breast cancer accounted for $34.1 \%$ of all female cancer cases. Breast cancer is the leading cause of cancer death in Malaysia with total of 21,634 cases of female breast cancer was diagnosed for the period of 2012-2016 (Azizah, 2019). Hence, it is compulsory to conduct research to understand the pathogenesis of breast cancer and discover the targeted therapy for the detection and treatment of breast cancer.

Estrogen hormone is important in normal mammary cells to regulate growth, differentiation and maintain homeostasis. Estrogen can acts as a mitogen that stimulates breast tissue to mitosis, and certain metabolites of estrogen also can act as carcinogens or genotoxin that directly damaging DNA, thereby causing cancer cells to form
(Cavalieri and Rogan, 2011). However, the effects of estrogen hormone alone do not fully lead to breast cancer development. Breast cancer tumors are dependent on estrogen and/or progesterone hormones for growth and this effect is mediated through estrogen receptor (ER) and progesterone receptor (PgR). Human epidermal growth factor receptor-2 (HER2/neu) belongs to a family of four homologous receptors involved in the tyrosine kinase mediated regulation of normal breast tissue growth and development (Iqbal and Iqbal, 2014). The high expression of HER2/neu in breast cancer results indicates of more aggressive tumor with a poor prognosis. Hormone receptor studies such ER, PgR, and HER2/neu are routinely done in breast carcinoma. It not only helps in the prognosis of the tumor but also helps in deciding the best treatment.

In order to understand the biology of cancer and develop cancer prevention strategies, chemically induced carcinogenesis models in rat are widely used. There are several types of carcinogen used to induce cancer in an animal model such as 1,2 dimethylbenz (a)-anthracene(DMBA), Diethylnitrosoamine (DEN), 
Azoxymethane (AOM), and N-Nitroso-N-Methylurea (NMU). NMU is administrated intraperitoneally to animals to induce the oncogenesis of the mammary ducts and yields a high incidence of estrogen and/or progesterone receptor (ER/PgR)-positive mammary tumors. NMUinduced mammary carcinoma is age dependent; and the model is widely used to screen and evaluate the potency of cancer-suppressing and promoting agents.

Sirolimus, also known as Rapamycin is a natural macrocyclic lactone produced by the bacterium Streptomyces hygroscopicus, with immunosuppressant properties (Martel et al., 1977). It was initially developed as an antifungal agent until it was discovered to have effective immunosuppressive and anti-proliferative properties due to its ability to inhibit mammalian target of rapamycin (mTOR) (Li et al., 2014). Sirolimus is a mammalian target of rapamycin inhibitor that has been shown to inhibit rather than promote cancers in experimental models. Sirolimus target mTORC1. Blocking of mTORC1 will inhibit cell growth factors, nutrients, energy and oxygen status supply that are required for cell growth and proliferation. However, long-term exposure to sirolimus will inhibit mTORC 2 by isolating newly synthesized mTOR molecules (Guduru and Arya, 2017).

Sunitinib (Sutent) is a tyrosine kinase inhibitor (TKI) indicated for first-generation multi-targeted ATP-competitive TKIs including the vascular endothelial growth factor receptors (VEGFRs) types 1 and 2 (FLT1 and FLK1/KDR), the platelet-derived growth factor receptors (PDGFR- $\alpha$ and PDGFR- $\beta$ ), the Fms Related Receptor Tyrosine Kinase (FLT3), Rearranged during Transfection (RET) kinases, and the stem cell factor receptor c-Kit. The VEGF family are frequently overexpressed in various solid tumors including mammary tumor and bind to vascular endothelium to induce angiogenesis. Inhibiting these tyrosine kinase receptors will block downstream signal transduction, thus affecting tumor angiogenesis and growth. Its antiangiogenic properties are well established and approved by Food and Drug Administration (FDA) in the use against treatment of gastrointestinal stromal tumor (GIST) after disease progression on or in tolerance to imatinib mesylate (Lopes and Bacchi, 2010), advanced renal cell carcinoma (RCC) (Adams and Leggas, 2007), adjuvant treatment of adult patients at high risk of recurrent RCC following nephrectomy (Fadil Hassan, 2018), pancreatic neuroendocrine tumors (pNET) in patients with not resectable locally advanced or metastatic disease (Delbaldo, Faivre, Dreyer, and Raymond, 2012) and hepatocellular carcinoma (HCC) (Zhu et al., 2009).

On the basis of these data, we therefore sought to explore the effects of sirolimus and sunitinib treated of NMU induced tumors on the tumor growth, histological changes, and protein expressions of ER, $P g R$ and HER2.

\section{Materials and Methods}

\section{Animal procedures}

Thirty-two female of Sprague Dawley rats were acquired from the Animal Research and Service Centre (ARASC), USM. The rats were then caged in environmentally controlled conditions (temperature 23 $\pm 2^{\circ} \mathrm{C}$, relative humidity $70 \pm 5 \%$, and alternate $14 \mathrm{~h}$ light $10 \mathrm{~h}$ dark cycle). They were fed with food pellets and tap water ad libitum. The principles in the care and use of laboratory animals were strictly in accordance with USM animal ethics guidelines and with supervision and husbandry facilities provided by ARASC (USM/ IACUC/2017/(108)(876).

\section{NMU, Sirolimus and Sunitinib preparation}

N-Methyl-N-Nitroso Urea (Cat. No. M325815) was provided by Toronto Research Chemicals, Canada. The off-white wet solid of NMU was freshly-prepared prior to injection by dissolved in $70 \mathrm{mg} / \mathrm{kg}$ body weight NMU with $0.9 \%$ normal saline followed by gentle heating in water bath and vigorous shaking. Both sirolimus and sunitinib were prepared by dissolving the powder in $10 \%$ DMSO, 40\% PEG300, 5\% PEG (80) and 0.9\% normal saline to make up $1 \mathrm{ml}$ solution to a final concentration of $10 \mathrm{mg} / 0.2 \mathrm{ml}$, while Sunitinib was dissolved in normal saline to the final concentration of $5 \mathrm{mg} / 0.2 \mathrm{ml}$.

\section{Tumor Induction and Detection}

The NMU at a dose of $70 \mathrm{mg} / \mathrm{kg}$ body weight was injected intraperitoneally two times. The first NMU injection was administrated when the rat's age were 21 days old, followed by second injection at the alternate days. The rats were weighed and palpated twice a week for the detection of mammary tumors. The mammary lesions growths were observed and their diameter size was measured by using Vernier calliper. The symptoms of illness or side effects which may cause by NMU toxicity were also observed.

\section{Experimental design}

All rats were randomly assigned into four groups. Group $1(n=8)$ served as a control group and was sacrificed after 5 days injection with physiological normal saline (used as a placebo) at size of $14.5 \pm 0.5 \mathrm{~mm}$. For the treated groups, the rats were anesthetized by Ketamine/ Xylazine. Then, the rats in Group $2(n=8)$ were treated with sirolimus, Group $3(\mathrm{n}=8)$ with sunitinib, and Group $4(\mathrm{n}=8)$ with sirolimus + sunitinib via an intralesional injection when the lesions reached diameter size of $14.5 \pm 0.5 \mathrm{~mm}$ twice for alternate days. The solution was freshly prepared prior to injection and kept on ice until intervention process. The rats in Group 2, 3 and 4 were sacrificed when the lesions regressed post 5 days intervention.

\section{Tumor sample collection}

All tumor-bearing rats were sacrificed by euthanized through exposure to carbon dioxide gaseous in a closed chamber. At necropsy, all grossly visible mammary tumors and normal breast pad were removed. A portion of each sample was fixed at room temperature in $10 \%$ normal buffered formalin while the remaining was fixed in RNA later solution. Tumor tissues were fixed in $10 \%$ normal buffered formalin for 24 hours at room temperature to obtain adequate fixation and optimal histology outcomes. The tissues were processed and embedded in paraffin for routine histological evaluation. Then, the paraffin 
sections of all tissues excised at necropsy were stained with Hematoxylin and Eosin ( $\mathrm{H}$ and $\mathrm{E})$.

\section{Immunohistochemistry}

Briefly, sections were deparaffinized, hydrated, and blocked for endogenous peroxidase. Heat-induced epitope retrieval was performed for all antibodies. Detailed staining protocols for all antibodies are listed in Table 1.

For ER and PgR protein, the nucleus staining of epithelial tumor tissues were evaluated using Allred scoring method. As per the Allred score for ER and PgR nuclear positivity, the proportion score (PS) (0-5) and the $\%$ positive tumor cells are respectively, $0(0 \%), 1(<1 \%)$, $2(1-10 \%), 3(11-33 \%), 4(34-66 \%)$, and $5(67-100 \%)$. The intensity of staining (IS) for the nuclear positivity of the cells graded as $0,1,2$, and 3 was as none, mild, moderate, and strong, respectively. The total scores for ER and PgR are given as TS $=$ PS + IS. TS 0 and 2 are negative scores, and $3,4,5,6,7$, and 8 are positive scores.

Membrane and/or cytoplasmic staining for HER2/neu antigen were scored using American Society of Clinical Oncology (ASCO)/College of American Pathologists (CAP) guideline. HER2/neu test result was positive for HER $2 /$ neu $3+$ based on circumferential membrane staining that is complete, intense. HER2/neu 2+ was equivocal based on circumferential membrane staining that is incomplete and/or weak/moderate and within $>10 \%$ of the invasive tumor cells; or complete and circumferential membrane staining that is intense and within $\leq 10 \%$ of the invasive tumor cells. HER $2 /$ neu test result is negative if; IHC $1+$ as defined by incomplete membrane staining that is faint/barely perceptible and within $>10 \%$ of the invasive tumor cells and; IHC 0 as defined by no staining observed or membrane staining that is incomplete and is faint/barely perceptible and within $\leq 10 \%$ of the invasive tumor cells.

\section{Statistical analysis}

Data was descriptively presented in mean \pm S.D or median \pm IQR and frequency (percentage). The differences in terms of immunohistochemistry expressions across all experimental groups were determined by the Kruskal-Wallis test with Bonferroni correction followed by Pairwise test for multiple comparisons. All statistical analyses were carried out using Statistical Product and Service Solutions (SPSS) version 26 (IBM, New York USA).

\section{Results}

Tumor incidence and latency

The sums of mammary tumor which were successfully

Table 1. Staining Protocol of IHC

\begin{tabular}{lccc}
\hline Primary antibody & Dilution & Antigen retrieval & Blocking \\
\hline $\begin{array}{l}\text { Estrogen receptor } \\
\text { (ER) }\end{array}$ & $1: 100$ & Tris-EDTA (pH 9) & 15 minutes \\
$\begin{array}{l}\text { Progesterone } \\
\text { receptor (PgR) }\end{array}$ & $1: 200$ & Citrate buffer (pH6) & 15 minutes \\
HER2/neu & $1: 100$ & Tris-EDTA (pH 9) & 15 minutes \\
\hline
\end{tabular}

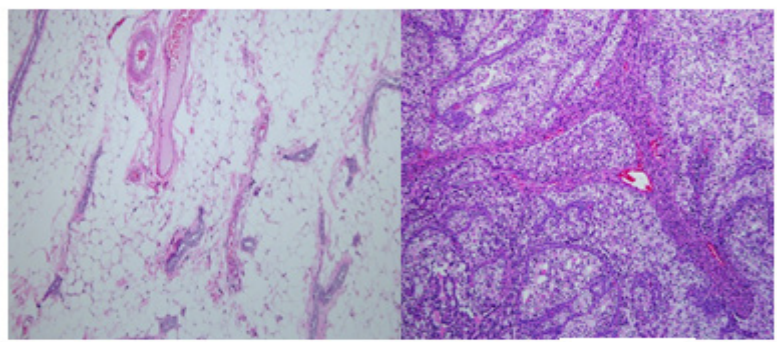

(i)

(ii)

Figure 1. Comparison of (i) Normal mammary gland of female Sprague-Dawley rat, and (ii) NMU-induced Invasive Breast Carcinoma. H\&E staining magnification $100 X$.

Table 2. Tumor Diameter (Mean \pm S.D) of Intervention Groups after First Treatment and Five Days Post Second Treatment

\begin{tabular}{|c|c|c|}
\hline \multirow[t]{2}{*}{ Groups } & \multicolumn{2}{|c|}{ Tumor diameter (Mean \pm S.D), mm } \\
\hline & $\begin{array}{l}\text { After first } \\
\text { treatment }\end{array}$ & $\begin{array}{l}\text { Five days after } \\
\text { second treatment }\end{array}$ \\
\hline Sirolimus & $11.3 \pm 4.0$ & $6.3 \pm 3.0$ \\
\hline Sunitinib & $12.5 \pm 2.2$ & $14.2 \pm 2.5$ \\
\hline Sirolimus + Sunitinib & $12.6 \pm 3.2$ & $11.2 \pm 2.4$ \\
\hline
\end{tabular}

excised from the rats were 35 tumors. Among them, 19 tumors $(54.3 \%)$ were observed to be located in the abdominal inguinal region while 16 tumors (45.7\%) located in cervical thoracic region of mammary gland chain. At macroscopic, they were solid and receiving blood supply from collateral vessels.

\section{Tumor diameter progression}

The tumor diameter in sunitinib treated group regressed after first injection to $12.5 \pm 2.2 \mathrm{~mm}$. However, five days after second treatment intervention, the tumor diameter increased to $14.2 \pm 2.5 \mathrm{~mm}$. In sirolimus treated group, the tumor diameter significantly regressed after treatment to $6.3 \pm 3.0 \mathrm{~mm}$. In sirolimus + sunitinib treated group, the tumor diameter also regressed to $11.2 \pm 2.4 \mathrm{~mm}$ after five days intervention of second treatment (Table 2).

\section{Histological Analysis}

In control group, a total of $8 \%$ of NMU mammary tumors were observed to show less aggressive Invasive Breast Carcinoma (IBC) cribriform subtype whilst the remainder $92 \%$ was aggressive subtypes (IBC papillary and IBC-NST). However, no benign mammary tumor was observed. Figure 1 showed comparison of histological

Table 3. The Tumor Types in the Intervention Groups

\begin{tabular}{lccc}
\hline Groups & \multicolumn{3}{c}{ Invasive Carcinoma } \\
& Cribriform (\%) & Papillary (\%) & $\begin{array}{c}\text { No Special } \\
\text { Type- NST (\%) }\end{array}$ \\
\hline Control & $1 / 16(6.25 \%)$ & $9 / 16(56.25 \%)$ & $6 / 16(37.5 \%)$ \\
Sirolimus & $9 / 16(56.25 \%)$ & $3 / 16(18.75 \%)$ & $4 / 16(25 \%)$ \\
Sunitinib & $7 / 16(44 \%)$ & $9 / 16(56 \%)$ & $0 / 16(0 \%)$ \\
Sirolimus + & $5 / 16(31.25 \%)$ & $9 / 16(56.25 \%)$ & $2 / 16(12.5 \%)$ \\
Sunitinib & & & \\
\hline
\end{tabular}

Asian Pacific Journal of Cancer Prevention, Vol 212921 


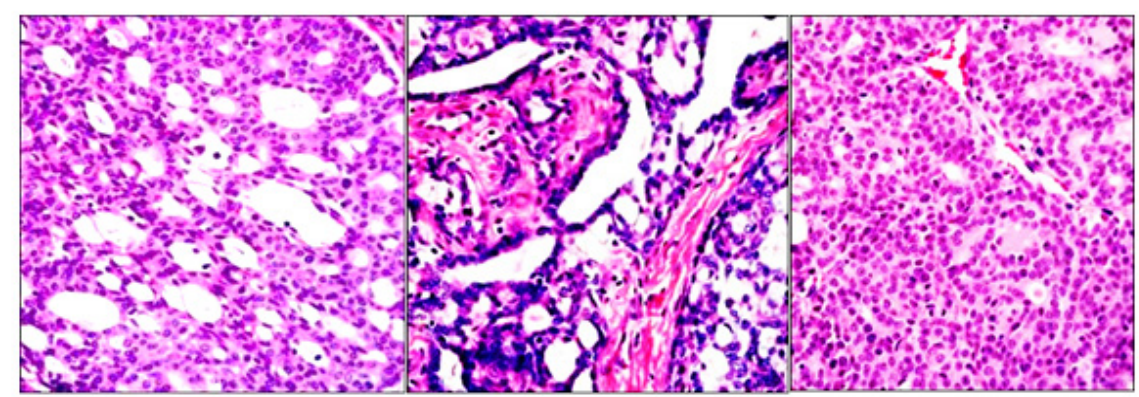

(A)

(B)

(C)

Figure 2. (A) NMU-induced Invasive Carcinoma of Cribriform. Characterized by well-defined spaces formed by arches of cells, tumor cells are small, moderate nuclear pleomorphism with no / rare mitotic figures. (B) NMU-induced Invasive Carcinoma of Papillary. The tumor has numerous papillary projections with thin fibrovascular core. (C) NMU-induced Invasive Carcinoma of No Special Type. The carcinoma displayed diffuses infiltration of neoplastic cells, with less tubule formation, highly nuclear pleomorphism and high mitotic rate. H\&E staining magnification 400x.

Table 4. Kruskal-Wallis Test Table for Total Score of ER, PgR, and HER2/neu

\begin{tabular}{|c|c|c|c|c|c|c|c|c|c|}
\hline \multirow[t]{2}{*}{ Group } & \multicolumn{3}{|c|}{ ER } & \multicolumn{3}{|c|}{$\mathrm{PgR}$} & \multicolumn{3}{|c|}{ HER2/neu } \\
\hline & $\begin{array}{c}\text { Median } \\
\text { (IQR) }\end{array}$ & $\begin{array}{l}\text { Chi-square } \\
\text { (df) }\end{array}$ & $\begin{array}{c}p \\
\text { value }\end{array}$ & $\begin{array}{c}\text { Median } \\
\text { (IQR) }\end{array}$ & $\begin{array}{l}\text { Chi-square } \\
\text { (df) }\end{array}$ & $\begin{array}{c}p \\
\text { value }\end{array}$ & $\begin{array}{c}\text { Median } \\
\text { (IQR) }\end{array}$ & $\begin{array}{l}\text { Chi-square } \\
\text { (df) }\end{array}$ & $\begin{array}{c}p \\
\text { value }\end{array}$ \\
\hline Control & $6.5(1)$ & $29.353(3)$ & $0.000 *$ & $8.0(1)$ & $27.426(3)$ & $0.000 *$ & $1.0(1)$ & $8.142(3)$ & $0.043 *$ \\
\hline Sirolimus & $4.0(1)$ & & & $5.0(2)$ & & & $1.0(1)$ & & \\
\hline Sunitinib & $5.0(1)$ & & & $7.0(1)$ & & & $1.0(1)$ & & \\
\hline Sirolimus + Sunitinib & $5.0(1)$ & & & $6.0(2)$ & & & $1.0(1)$ & & \\
\hline
\end{tabular}

*significant value: $\mathrm{p}<0.05$

features of normal mammary gland with the malignant invasive carcinoma, and the histology of IBC of cribriform, papillary and NST were shown in Figure 2.

As shown in Table 3, the trend of Invasive Carcinoma pattern in tumor tissues for control group showed the highest papillary pattern (56.25\%). However, the Invasive Carcinoma of Papillary pattern decreased in Sirolimustreated group (18.75\%). The Sirolimus-treated group showed the highest less aggressive Invasive Carcinoma of Cribriform pattern (56.25\%). In contrast, the tumor tissues treated with sunitinib and sirolimus + sunitinib treated groups showed the highest pattern of papillary pattern (56\% and $56.25 \%$ respectively) compared to sirolimus-treated group.

\section{Immunohistochemistry}

The results of protein expressions of $E R, P g R$, and HER2/neu on tumor are shown in Figure 3 and the IHC scoring are summarized in Table 4 . The statistical analyses are shown in Table 5 and Table 6.

For Estrogen Receptor (ER), Kruskal-Wallis test

Table 5. The Expressions of ER, PgR, and HER2/neu between Intervention and Control Groups

\begin{tabular}{lccc}
\hline Group & p-value & & \\
& ER & PgR & HER2/neu \\
\hline Control vs Sirolimus & $0.000^{*}$ & $0.000^{*}$ & 0.446 \\
Control vs Sunitinib & 0.087 & 0.625 & 1 \\
Control vs Sirolimus + Sunitinib & $0.011^{*}$ & $0.038^{*}$ & 0.815 \\
\hline *, Pairwise Test; ${ }^{*}$, significant value: $\mathrm{p}<0.05$ & &
\end{tabular}

provided very strong evidence of a difference $(p=0.000)$ between the median ranks of at least one pair of groups. Pairwise tests were carried out for the four pairs of groups. There was very strong evidence of ER total score readings between sirolimus treated and sunitinib treated group ( $p=0.020$, adjusted using the Bonferroni correction), sirolimus treated and control group of a difference, $(p=0.000$, adjusted using the Bonferroni correction), and ER total score of control and combinational-treated group $(\mathrm{p}=0.011$, adjusted using the Bonferroni correction). The median ER total score for the control group was 6.5 compared to 4.0 in the sirolimus-treated group. There was no evidence of a difference between the other pairs.

Progesterone Receptor (PgR) Kruskal-Wallis test showed significant difference $(p=0.000)$ between the median ranks of at least one pair of groups. Pairwise tests were carried out for the four pairs of groups. There were significant differences in PgR total score readings between sirolimus treated and sunitinib treated group $(p=$ 0.003, adjusted using the Bonferroni correction), sirolimus

Table 6. The Expressions of ER, PgR, and HER2/neu amongst the Intervention Groups

\begin{tabular}{lccc}
\hline Group & p-value & & \\
& ER & PgR & HER2/neu \\
\hline Sirolimus vs Sunitinib & $0.020^{*}$ & $0.003 *$ & 0.093 \\
Sirolimus vs Sirolimus + Sunitinib & 0.142 & 0.109 & 1 \\
Sunitinib vs Sirolimus + Sunitinib & 1 & 1 & 0.2 \\
\hline *, Pairwise Test; ${ }^{*}$, significant value: $\mathrm{p}<0.05$ & &
\end{tabular}


(A)

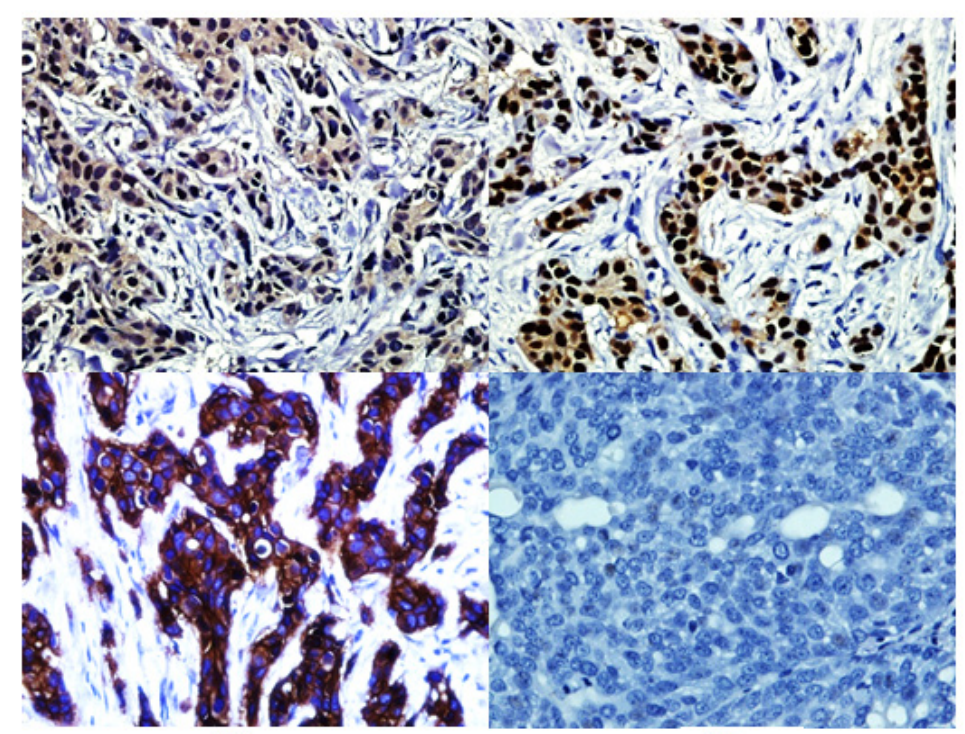

(C)
(D)

Figure 3. Immunohistochemistry Expressions of Representative Markers on Tumor Specimens. (A) Invasive Carcinoma positive control for ER, (B) Positive staining of PgR in Invasive Breast Carcinoma, (C) Positive control for HER2/neu, (D) Negative staining of ER in Invasive Breast Carcinoma. (Original magnification x400)

treated and control group of a difference, $(p=0.000$, adjusted using the Bonferroni correction), and $\mathrm{PgR}$ total score of control and sirolimus + sunitinib treated group $(p=0.0038)$. The median PgR total score for the control group was 8.0 compared to 5.0 in the sirolimus-treated group. There was no evidence of a difference between the other pairs.

Kruskal-Wallis test for HER2/neu expression also provided a difference $(\mathrm{p}=0.043)$ between the median ranks of at least one pair of groups. However, the pairwise tests carried out for the four pairs of groups showed no strong evidence of HER2/neu total score readings between all four groups when the $p$-value for all comparisons is $>0.05$. The median for HER2/neu total score for all groups was 1. Hence, there was no evidence of difference between all pairs.

\section{Discussion}

In this study, we investigated the synergistic effects of sirolimus (anti-mTOR) and sunitinib (multi-targeted receptor tyrosine kinase inhibitors) on NMU-induced breast cancer. The novel findings in this study are: 1) Treatment with sirolimus alone lowered expressions of $E R$ and $P g R$ of breast cancer and reduced tumor size; 2) There is no significant difference in $E R$ and $P g R$ expressions between control tumor and Sunitinib treated tumor; 3 ) Sunitinib treated tumors reduce in diameter after the first treatment, but the diameter increases after the second treatment; 4) Sunitinib treated tumor does not show any aggressive invasive carcinoma of NST histological subtypes; 5) All NMU-induced tumors are HER2/neunegative scoring.

Breast cancer is dependent on estrogen and/or progesterone hormones for growth and this is mediated through estrogen receptors (ERs) and progesterone receptors (PgRs). ER specifically ER $\alpha$ contributes to tumor aggressiveness and the increasing histological grade (Muscat et al., 2013). As Booth and Smith, (2006) reported that ER and PgR are localized in the nucleus of epithelial cells and will co-upregulated to label-retaining mammary epithelial cells that divide asymmetrically and retain their template DNA strands. In this study, it was proved that anti-tumor treatment potentially preventing the estrogen dependent mechanism towards cancer cells progression and lowered the $E R$ and $P g R$ expression.

Our study disclosed that all malignant breast carcinoma of control tumor tissue are both overexpressed of ER and PgR. However, the ER and $P g R$ expressions of sirolimus-treated and sirolimus + sunitinib treated tumor are significantly lower compared to control group. In relation with tumor diameter, the sirolimus-treated and sirolimus + sunitinib treated tumor noticeably reduced the tumor size of NMU-induced breast cancer. These suggest that the treatment reduces the growth of premalignant rat mammary lesions and inhibits the malignant transformation of mammary carcinoma.

However, sunitinib does not reduce the expressions of $E R$ and $P g R$ expressions. This result may be supported with previous research conducted by Miller et al., (2010) that showed direct inhibition of PI3K/mTOR pathway effectively suppressed the growth of both estrogenindependent and -dependent cells breast cancer cell growth associated with hyperactivation of the IGF IR/InsR/PI3K/ mTOR pathway, but inhibition of nodes upstream (RTKs) and downstream (mTOR) of PI3K only partially blocked breast cancer cell growth.

Sunitinib has been used as anticancer treatments in several types of tumor including breast cancer, however previous clinical observations of sunitinib treatment showed that this therapy has limited efficacy. Researchers figured out that when sunitinib as anti-angiogenic agents 
administered on an intermittent schedule; 4 weeks on and 2 weeks off, tumor regrowth was seen during drug-free periods (Burstein et al., 2008) or upon discontinuation of the treatment (Mulet-Margalef and Garcia-DelMuro, 2016). This result was explained due to sunitinib inhibits primary tumor growth, but the inhibition is exceptional lasting responses and only show moderate increases in progression-free survival and little benefit in overall survival. This might relate to previous studies when sunitinib treated tumors reduce in diameter after first treatment, but the diameter increases after second treatment. Sunitinib might generate intratumoral hypoxia modulating the metastatic process (Lu and Kang, 2010) and stimulating cancer stem cells (CSC) (Seidel et al., 2010).

HER2/neu-positive breast cancer is a more aggressive type of breast cancer compared with HER2/neu-negative types. Our study showed all NMU-induced breast cancers are estrogen and progesterone receptors positive but HER2/neu negative (all luminal A subtype). In the present in-vivo study, all induced mammary tumors in female rats were adenocarcinomas (luminal A subtype) based on the results of the ER and/or PgR positivity and HER2/neu negativity, as in previous report (Kinoshita et al., 2016). The negative expressions of HER2/neu in our result may explain decreasing grade in aggressiveness of invasive carcinoma of NST of treated groups. In addition, sunitinib as tyrosine kinase inhibitor might not be able to work efficiently to inhibit HER2/neu which involved in the tyrosine kinase mediated regulation of mammary gland since HER2/neu does not well express in NMU-induced breast cancer.

In conclusion, treatment with sirolimus alone showed significant mammary tumor inhibition which presumably exerts its inhibitory effect through mTOR pathway. ER and PgR play a major role in breast cancer cell development and positively correlated with breast cancer cell proliferation. $E R$ and $P g R$ expressions of sirolimus treated and combination treated tumor are significantly lower compared to control group, hence evidently reduced tumor size of NMU-induced breast cancer. In contrast, treatment with sunitinib shrinks the solid tumor after first treatment, but the diameter increases after second treatment. This might due to sunitinib generate intratumoral hypoxia modulating the metastatic process and stimulating cancer stem cells in NMU-induced mammary tumor growth. Thus, present results suggested that sirolimus is not synergistic or additive with sunitinib. Furthermore, sunitinib might be an antagonist towards the sirolimus activity as multi-targeted tyrosine kinase inhibitor.

\section{Acknowledgements}

We would like to acknowledge the Pathology department and USM Animal Research and Service Centre (ARASC) staff for their support with laboratory supplies and for teaching us.

\section{Funding Statement}

This study was supported by the Research University grant account No: RUI/1001/PPSP/8012222 from
Universiti Sains Malaysia.

\section{References}

Adams VR, Leggas M (2007). Sunitinib Malate for the treatment of metastatic renal cell carcinoma and gastrointestinal stromal tumors. Clin Ther, 29, 1338-53.

Azizah AMHB, Nirmal K, Siti Zubaidah AR, et al (2019). Malaysia National Cancer Registry Report (MNCR) 20122016. (5). Ministry of Health Malaysia Retrieved from http:// nci.moh.gov.my.

Booth BW, Smith GH (2006). Estrogen receptor-alpha and progesterone receptor are expressed in label-retaining mammary epithelial cells that divide asymmetrically and Retain Their Template Dna Strands. Breast Cancer Res, 8, 49.

Bray F, Ferlay J, Soerjomataram H, et al (2018). GLOBAL CANCER STATISTICS 2018: GLOBOCAN estimates of incidence and mortality worldwide for 36 cancers in 185 countries. CA Cancer J Clin, 68, 394-424.

Burstein HJ, Elias AD, Rugo HS, et al (2008). Phase Ii sudy of sunitinib malate, An oral multitargeted tyrosine kinase inhibitor, In patients with metastatic breast cancer previously treated with an anthracycline and a Taxane. J Clin Oncol, 26, 1810-6.

Cavalieri EL, Rogan EG (2011). Unbalanced metabolism of endogenous estrogens in the etiology and prevention of human cancer. J Steroid Biochem Mol Biol, 125, 169-80.

Delbaldo C, Faivre S, Dreyer C, Raymond E (2012). Sunitinib in advanced pancreatic neuroendocrine tumors: Latest Evidence And Clinical Potential. Therap Adv Med Oncol, 4, 9-18.

Fadil Hassan SL, Kiran S, Anil K (2018). Current role of adjuvant therapy in high risk for recurrence resected kidney cancer. Intech Open.

Guduru SKR, Arya P (2017). Synthesis and biological evaluation of rapamycin-derived, next generation small molecules. Med Chem Comm, 9, 27-43.

Iqbal N (2014). Human epidermal growth factor receptor 2 (Her2) in cancers: Overexpression And Therapeutic Implications. Mol Biol Int, 2014, 852748.

Kinoshita Y, Yoshizawa K, Hamazaki K, et al (2016). Dietary effects of mead acid on N-Methyl-N-Nitrosourea-induced mammary cancers in female sprague-dawley rats. Biomed Rep, 4, 33-39.

Li J, Kim SG, Blenis J (2014). Rapamycin: One Drug, Many Effects. Cell Metabolism, 19, 373-9.

Lopes LF, Bacchi CE (2010). Imatinib treatment for gastrointestinal stromal tumor (Gist). J Cell Mol Med, 14, 42-50.

Lu X, Kang Y (2010). Hypoxia and hypoxia-inducible factors: Master Regulators Of Metastasis. Clin Cancer Res, 16, 5928.

Martel R, Klicius J, Galet S (1977). Inhibition of the immune response by Rapamycin, A New Antifungal Antibiotic. $C A$ $J$ Physiol Pharmacol, 55, 48-51.

Miller TW, Hennessy BT, Gonzalez-Angulo AM, et al (2010). Hyperactivation of phosphatidylinositol-3 kinase promotes escape from hormone dependence in estrogen receptorpositive human breast cancer. J Clin Invest, 120, 2406-13.

Mulet-Margalef N, Garcia-Del-Muro X (2016). Sunitinib In the treatment of gastrointestinal stromal tumor: Patient Selection And Perspectives. OncoTargets Ther, 9, 7573-82.

Muscat GE, Eriksson NA, Byth K, et al (2013). Research resource: Nuclear receptors as transcriptome: Discriminant and prognostic value in breast cancer. Mol Endocrinol, 27, 350-65.

Seidel S, Garvalov BK, Wirta V, et al (2010). A hypoxic niche 
regulates glioblastoma stem cells through hypoxia inducible factor $2 \alpha$. Brain, 133, 983-95.

Zhu AX, Duda DG, Sahani DV, Jain RK (2009). Development of sunitinib in hepatocellular carcinoma: Rationale, early clinical experience, and correlative studies. Cancer $J$ (Sudbury, Mass.), 15, 263-8.

\section{c) (i) (8)}

This work is licensed under a Creative Commons AttributionNon Commercial 4.0 International License. 